\title{
ENVELHECIMENTO ARTIFICIAL ACELERADO POR RADIAÇÃO ULTRAVIOLETA DE MADEIRAS DE Eucalyptus benthamii E Pinus caribaea var. hondurensis
}

\author{
Sabrina Andrade Martins ${ }^{1}$, Cristiane Moreira Tavares dos Santos ${ }^{2}$, Joaquim Carlos Gonçalez ${ }^{3}$, \\ José Arlete Alves Camargos ${ }^{4}$ \\ ${ }^{1}$ Tecnóloga da Madeira, Mestranda em Ciências Florestais, UnB, Brasília, DF, Brasil - sabrinaand@ gmail.com \\ ${ }^{2}$ Eng ${ }^{a}$ Florestal, Mestranda em Ciências Florestais, UnB, Brasília, DF, Brasil - crismtsantos@yahoo.com.br \\ ${ }^{3}$ Eng. Florestal, Dr., Depto. de Engenharia Florestal, UnB, Brasília, DF, Brasil - goncalez@unb.br \\ ${ }^{4}$ Administrador, M.Sc., LPF/IBAMA, Brasília, DF, Brasil - camargos@lpf.ibama.gov.br \\ Recebido para publicação: 10/10/2009 - Aceito para publicação: 16/03/2010
}

\begin{abstract}
Resumo
Este trabalho teve como objetivo avaliar os processos de fotodegradação da cor superficial de duas espécies de madeiras, Pinus caribaea var. hondurensis e Eucalyptus benthamii, ao longo do tempo de exposição à radiação ultravioleta em um reator fotoquímico. As amostras de $P$. caribaea var. hondurensis foram avaliadas na face radial, enquanto que as de Eucalyptus benthamii na face tangencial. As medidas dos parâmetros colorimétricos, segundo o Sistema CIELAB 1976 (L*, a*, b*, C, e h*), foram obtidas por meio de um espectrofotocolorímetro, em intervalos de tempo de $0,42,84,126$ e 168 horas. Ambas as espécies apresentaram um escurecimento ao longo do tempo, principalmente nas primeiras horas de exposição. A madeira de E. benthamii apresentou uma maior variação de cor $(\Delta \mathrm{E}=19,72)$ que a madeira de $P$. caribaea var. hondurensis $(\Delta \mathrm{E}=17,82)$ após as 168 horas.

Palavras-chave: Envelhecimento acelerado; colorimetria; madeira; eucalipto; pínus.
\end{abstract}

\begin{abstract}
Accelerated artificial aging by ultraviolet irradiation of Eucalyptus benthamii and Pinus caribaea var. hondurensis woods. The main objective of this research was to study the photo degradation process of superficial color of two wood species, Pinus caribaea var. hondurensis and Eucalyptus benthamii, over ultraviolet irradiation exposure time in a photochemical reactor. Pinus caribaea var. hondurensis samples were evaluated in the radial plane, while Eucalyptus benthamii samples were in tangential plane. The colorimetric parameters measures under the CIELAB 1976 system (L*, a*, b*, $\mathrm{C}, \mathrm{e} \mathrm{h*)}$ were obtained using a Spectophotometer, in the following time intervals: $0,42,84,126$ and 168 hours. Both species presented darkening over time, especially in the first hours of exposure. The $E$. benthamii wood had more changes in its color parameters $(\Delta \mathrm{E}=19.72)$ than $P$. caribaea var. hondurensis wood $(\Delta \mathrm{E}=17.82)$ after 168 hours.

Keywords: Accelerated aging; colorimetry; wood; eucalyptus; pine.
\end{abstract}

\section{INTRODUÇÃO}

A caracterização tecnológica da madeira vai além dos estudos anatômicos físicos e mecânicos. Outros parâmetros, como a cor, os constituintes químicos, a secagem e a durabilidade natural, dentre outros, completam uma melhor caracterização e utilização de uma determinada espécie madeireira. Entretanto esses parâmetros são menos utilizados, talvez pela maior dificuldade da realização desses ensaios (GONÇALEZ et al., 2001).

De acordo com Camargos; Gonçalez (2001), a cor é uma das características mais importantes para a identificação e indicação de usos de espécies de madeira, principalmente quando associada aos aspectos de textura e desenho.

A cor de uma madeira não é estável, podendo alterar-se com o passar do tempo, com frequência escurecendo, em função da oxidação causada sobretudo pela luz, que reage com componentes químicos, 
principalmente extrativos e lignina. Os extrativos, mesmo em pequenas quantidades, têm grande importância na determinação da cor da madeira (TSOUMIS, 1991).

Assim, sempre que exposta à ação de intempéries, a cor e a textura da madeira são alteradas (SUDIYANI et al., 1999). Essas alterações ocorrem devido à ação dos raios ultravioletas, que provocam tanto a fotodegradação quanto a fotodescoloração da madeira, diminuindo seu tempo de vida útil (GOUVEIA, 2008).

Segundo Creemers et al. (2002), todos os elementos do clima influenciam mutuamente os dados sobre a madeira, mas a radiação solar e a precipitação são dominantes em relação aos outros mecanismos de intemperismo.

Embora a ação do intemperismo seja superficial, constitui um problema para os usuários de madeira, pois afeta tanto a sua aparência estética como o desempenho dos produtos de proteção aplicados sobre ela. Essas alterações podem ocorrer em função de diversos fatores, mas a ação dos raios ultravioletas, que provocam a fotodescoloração da madeira, diminuindo a sua vida útil, é um dos mais importantes.

A fotodegradação ocorre somente na superfície da madeira, pois, após as primeiras reações, é formada uma pequena camada que protegerá as camadas inferiores e os outros constituintes celulósicos. Registros históricos indicam que, mesmo após 100 anos de exposição contínua à luz, a madeira é erodida em não mais que sete milímetros (CHANG et al., 1982).

Segundo Temiz et al. (2005), a descoloração da madeira exposta à luz solar ocorre devido à modificação de grupos cromóforos capazes de absorver a radiação ultravioleta no comprimento de onda entre 300 e $400 \mathrm{~nm}$.

A lignina é o principal constituinte responsável pela fotodegradação da madeira. Sua estrutura fenólica possui grupos cromóforos que sofrem a ação dos raios ultravioletas. Os mecanismos de fotodegradação da lignina são complexos e geram diversos radicais fenólicos, sendo o principal deles o guaiacol. Esse radical é transformado em estruturas quinoides, que são a origem do amarelecimento da superfície da madeira (GEORGE et al., 2005). Como as coníferas normalmente possuem teores maiores de lignina, as madeiras de folhosas são consideradas mais resistentes à mudança de cor do que as madeiras de coníferas (MITSUI; TSUCHIKAWA, 2005).

As reações fotoquímicas que ocorrem na lignina são as principais causas de degradação, devido à incidência de luz na madeira. Grupos como $\alpha$-carbonil, bifenil, anéis de dupla ligação conjugada, podem absorver a radiação ultravioleta e formar grupos cromóforos, como as carbonilas e carboxilas, estruturas responsáveis pela alteração de cor nas madeiras (AYADI et al., 2003).

Muitas pesquisas têm sido desenvolvidas na tentativa de encontrar soluções para a melhoria das propriedades da madeira, sobretudo para otimizar as suas características quanto ao seu uso. O estudo do comportamento colorimétrico da madeira perante a ação de radiação ultravioleta é uma forma de contribuir para o conhecimento dessa matéria-prima, na busca de alternativas para prolongamento de sua vida útil.

Diante do exposto, a hipótese principal do trabalho é que o envelhecimento acelerado da madeira, por meio de radiação ultravioleta, ocasiona modificações em sua coloração. O sistema CIELAB se apresenta como uma ferramenta que pode detectar essas modificações de forma rápida e precisa.

O objetivo deste trabalho foi avaliar o efeito da radiação ultravioleta, através do envelhecimento artificial acelerado, na coloração da madeira de E. benthamii e $P$. caribaea var. hondurensis.

\section{MATERIAL E MÉTODOS}

A pesquisa foi realizada na Universidade de Brasília (UnB) e no Laboratório de Produtos Florestais (LPF) do Serviço Florestal Brasileiro.

A madeira de E. benthamii é proveniente de plantio experimental localizado na cidade de Otacílio Costa, SC, e a madeira de P. caribaea var. hondurensis foi adquirida no mercado local de Brasília, DF. Para cada espécie foram confeccionadas 4 amostras, nas dimensões de $8,0 \mathrm{~cm}$ de comprimento, $3,0 \mathrm{~cm}$ de largura e $0,5 \mathrm{~cm}$ de espessura, retiradas no sentido tangencial no E. benthamii e no sentido radial no $P$. caribaea var. hondurensis.

Para cada corpo de prova foram feitas 15 medições dos parâmetros colorimétricos ( $\mathrm{L}^{*}, \mathrm{a}^{*}, \mathrm{~b}^{*}, \mathrm{C}$ e $\mathrm{h}^{*}$ ), distribuídas de forma homogênea na amostra. As medições realizadas antes de serem colocadas no 
envelhecimento superficial acelerado serviram como testemunhas. Após, as amostras foram colocadas no reator, para receberem a radiação ultravioleta. As amostras eram retiradas e tinham suas medições colorimétricas realizadas a cada 42 horas. Esse procedimento foi executado por 4 vezes $(42 \mathrm{~h}, 84 \mathrm{~h}, 126 \mathrm{~h}$ e $168 \mathrm{~h}$ ), resultando em 168 horas de fotodegradação e 5 medidas colorimétricas.

De acordo com Chang; Chang (2001), o envelhecimento provocado em amostras de três espécies de coníferas (western hemlock, china fir, japanese cedar) irradiadas por lâmpadas ultravioletas UVA-351, é 250 vezes maior que o ocorrido em ambiente interno.

As medidas dos parâmetros colorimétricos $\left(\mathrm{L}^{*}, \mathrm{a}^{*}, \mathrm{~b}^{*}, \mathrm{C}\right.$ e $\left.\mathrm{h}^{*}\right)$ foram feitas em um espectrofotocolorímetro Datacolor Microflash 200d conectado a um microcomputador, com o iluminante D65 e ângulo standart de $10^{\circ}$ (Figura 1).

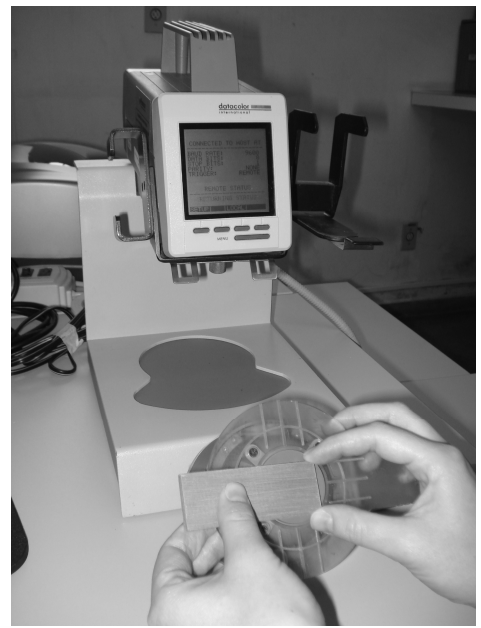

Figura 1. Medição realizada em espectrofotocolorímetro.

Figure 1. Measurement held in spectophotometer.

Para simular o efeito da radiação ultravioleta na superfície da madeira nas amostras, utilizou-se um reator fotoquímico Rayonet com 12 lâmpadas UV, cada uma com $8 \mathrm{~W}$, as quais emitem radiação no comprimento de onda de 350 nanômetros. As amostras foram fixadas em um carrossel, de forma que as faces analisadas ficaram expostas a aproximadamente dois centímetros de distância das lâmpadas (Figura 2).

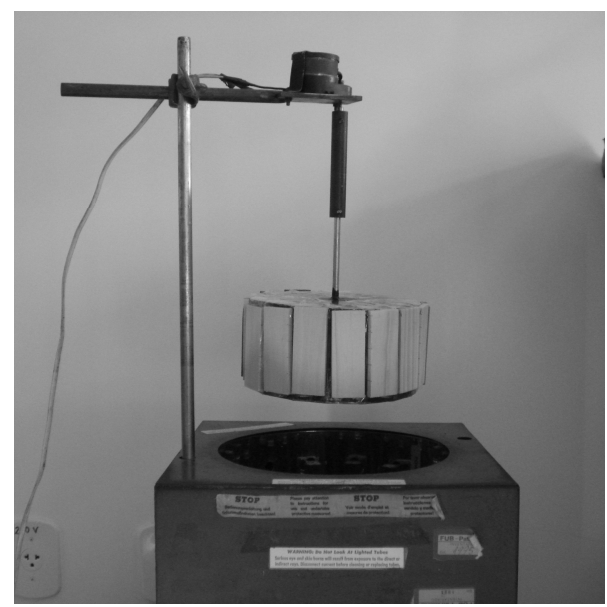

Figura 2. Amostras antes de serem colocadas dentro do reator.

Figure 2. Samples before placement into reactor. 
A análise da alteração na cor da madeira antes e após os tratamentos $(\Delta \mathrm{E})$ foi realizada pela metodologia adotada por Mitsui; Tsuchikawa (2005), calculada através da diferença entre as coordenadas obtidas das amostras antes e após o procedimento de envelhecimento, conforme a equação 1 .

$$
\Delta E=\sqrt{\Delta L^{2}+\Delta a^{2}+\Delta b^{2}}
$$

Em que: $\mathrm{L}^{*}$ : claridade;

$a^{*}$ : matizes do eixo vermelho $(+a)-$ verde $(-a)$;

$b^{*}$ : matizes do eixo amarelo $(+b)-$ azul $(-b)$.

Para a análise estatística, foi utilizado o programa Genes (aplicativo computacional em genética e estatística) e análise de variância (ANOVA) com decomposição em componentes ortogonais, com a qual foi avaliada a alteração de cada parâmetro colorimétrico ( $\mathrm{L}^{*}, \mathrm{a}^{*}, \mathrm{~b}^{*}, \mathrm{C}$ e $\left.\mathrm{h}^{*}\right)$ ao longo do tempo em exposição à radiação ultravioleta.

\section{RESULTADOS E DISCUSSÃO}

A tabela 1 mostra os valores médios dos parâmetros colorimétricos $\left(\mathrm{L}^{*}, \mathrm{a}^{*}, \mathrm{~b}^{*}, \mathrm{C}\right.$ e $\left.\mathrm{h}^{*}\right)$ para as espécies de E. benthamii e $P$. caribaea var. hondurensis, obtidos para as testemunhas e a cada intervalo de 42 horas de fotodegradação. Cada valor representa a média de 60 medidas (4 amostras com 15 repetições cada).

Tabela 1. Médias e desvio padrão (DP) das variáveis colorimétricas para as espécies de Eucalyptus benthamii e de Pinus caribaea var. hondurensis.

Table 1. Averages and standard deviation of colorimetric variables for Eucalyptus benthamii and Pinus caribaea var. hondurensis.

\begin{tabular}{|c|c|c|c|c|c|c|c|c|c|c|}
\hline $\begin{array}{l}\text { Tempo } \\
\text { (horas) }\end{array}$ & $\mathbf{L}^{*}$ & DP & $\mathbf{a}^{*}$ & DP & $\mathbf{b}^{*}$ & DP & C & DP & $\mathbf{h}^{*}$ & DP \\
\hline \multicolumn{11}{|c|}{ Eucalyptus benthamii } \\
\hline 0 & 78,46 & 0,50 & 10,83 & 0,45 & 18,66 & 0,57 & 21,59 & 0,41 & 59,85 & 1,58 \\
\hline 42 & 68,94 & 0,45 & 15,66 & 0,29 & 28,72 & 0,59 & 32,71 & 0,61 & 61,40 & 0,42 \\
\hline 84 & 65,68 & 0,38 & 16,10 & 0,35 & 29,74 & 0,49 & 33,82 & 0,58 & 61,58 & 0,25 \\
\hline 126 & 63,58 & 0,81 & 15,99 & 0,45 & 29,68 & 0,52 & 33,71 & 0,67 & 61,69 & 0,32 \\
\hline 168 & 62,62 & 0,69 & 15,76 & 0,48 & 29,33 & 0,47 & 33,30 & 0,62 & 61,76 & 0,41 \\
\hline \multicolumn{11}{|c|}{ Pinus caribaea var. hondurensis } \\
\hline 0 & 81,00 & 1,45 & 6,18 & 0,65 & 26,60 & 0,91 & 27,31 & 1,02 & 76,96 & 0,96 \\
\hline 42 & 75,58 & 1,24 & 9,15 & 0,53 & 35,28 & 0,53 & 36,45 & 0,59 & 75,47 & 0,72 \\
\hline 84 & 73,12 & 1,06 & 10,62 & 0,43 & 37,59 & 0,44 & 39,06 & 0,49 & 74,22 & 0,55 \\
\hline 126 & 71,20 & 1,07 & 12,05 & 0,42 & 38,86 & 0,62 & 40,69 & 0,63 & 72,77 & 0,58 \\
\hline 168 & 69,95 & 0,92 & 12,55 & 0,36 & 39,04 & 0,43 & 41,00 & 0,44 & 72,18 & 0,48 \\
\hline
\end{tabular}

Observando os desvios padrões apresentados na tabela 1 , pode-se inferir que a madeira de $E$. benthamii apresentou uma coloração mais homogênea que a madeira de P. caribaea var. hondurensis. A maior homogeneidade do E. benthamii pode ser explicada pelo seu corte estar apresentado na face tangencial, enquanto que a de $P$. caribaea var. hondurensis apresentava diferenças de coloração do lenho inicial e lenho tardio em sua face radial.

Tomando-se a tabela de cor sugerida por Camargos (1999), que considera o sistema CIELAB 1976, as madeiras de E. benthamii e de P. caribaea var. hondurensis em estado natural (testemunha) podem ser classificadas, respectivamente, como rosa-acinzentado e amarelo-claro.

Verifica-se ainda que há uma tendência de escurecimento nas duas espécies com o aumento da exposição à radiação ultravioleta. Esse escurecimento pode ser explicado quantitativamente pela diminuição dos valores da claridade $\left(\mathrm{L}^{*}\right)$ e pelo aumento dos valores da coordenada $\mathrm{a}^{*}$, que é o parâmetro responsável pela coloração vermelha. No caso do P. caribaea var. hondurensis, além da coordenada a*, a 
coordenada $\mathrm{b}^{*}$ tem presença marcante, ajudando a compor a cor da madeira mais escurecida, dando o tom "escuro-amarelado" para essa espécie.

Foi realizada, para cada parâmetro colorimétrico, em cada uma das espécies, a análise de variância (ANOVA), com decomposição em componentes ortogonais, permitindo assim a determinação do melhor modelo de alteração dos parâmetros em relação ao tempo de exposição à radiação ultravioleta.

A relação que se mostrou mais adequada para o parâmetro claridade $\left(L^{*}\right)$ nas duas espécies estudadas foi a de um modelo quadrático, com $\mathrm{R}^{2}$ de 0,98 para o $E$. benthamii e de 0,91 para o $P$. caribaea var. hondurensis (Figura 3). Os valores de $\mathrm{L}^{*}$ diminuíram principalmente nas primeiras horas, tendendo a uma estabilização após períodos mais longos de exposição.

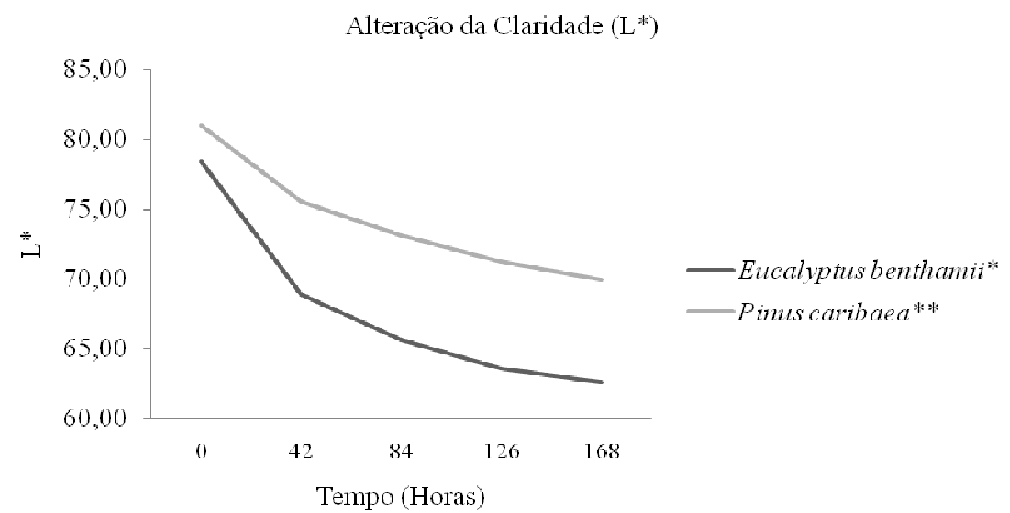

$* \mathrm{~L}=77,87-0,21 \times \mathrm{T}+0,00074 \times \mathrm{T}^{2}, \mathrm{R}^{2}$ de 0,98 . Significativo a $1 \%$ (E. benthamii)

$* * \mathrm{~L}=80,74-0,12 \times \mathrm{T}+0,00036 \times \mathrm{T}^{2}, \mathrm{R}^{2}$ de 0,91 . Significativo a $1 \%$ (P. caribaea var. hondurensis).

Figura 3. Alteração da claridade ( $\left.\mathrm{L}^{*}\right)$ da madeira ao longo do tempo.

Figure 3. Change of wood lightness ( $\left.\mathrm{L}^{*}\right)$ over time.

Para as coordenadas a* e b*, a relação que se mostrou mais adequada para a madeira de $P$. caribaea var. hondurensis foi a do modelo quadrático, com o $\mathrm{R}^{2}$ de 0,95 para ambas as variáveis, enquanto que para a madeira de $E$. benthamii a relação mais adequada para as variáveis $\mathrm{a}^{*} \mathrm{e} \mathrm{b}^{*}$ foi a do modelo cúbico, com o $\mathrm{R}^{2}$ de 0,95 e 0,98, respectivamente (Figuras 4 e 5).

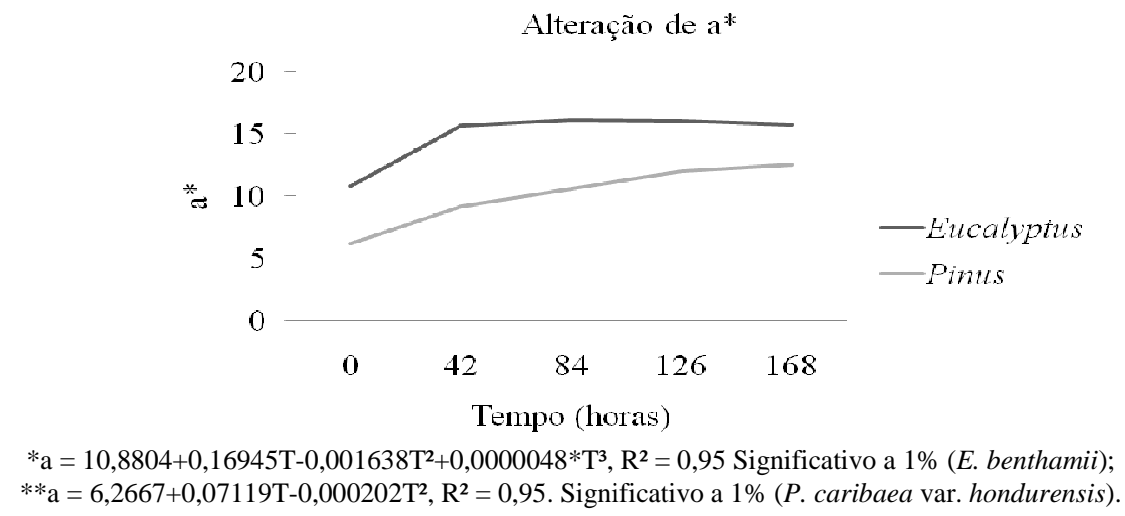

Figura 4. Alteração da coordenada $\mathrm{a}^{*}$ em função da fotodegradação da madeira.

Figure 4. Change of coordinate $\mathrm{a}^{*}$ according to photo degradation of wood.

Analisando-se a figura 4, percebe-se que os valores médios de a* são maiores para o $E$. benthamii, conferindo a presença mais marcante da coloração vermelha para essa espécie. Observa-se também que o comportamento da variável $a^{*}$ na madeira de $E$. benthamii teve um aumento mais pronunciado nas primeiras 42 horas de radiação e depois mostrou uma tendência de estabilização nas horas seguintes. Ou seja, a madeira dessa espécie, nas primeiras horas de radiação ultravioleta, escurece 
fortemente, vindo praticamente a estabilizar sua cor depois de 42 horas de tratamento. Já para a madeira de P. caribaea var. hondurensis, essa mesma variável apresentou um aumento moderado e constante durante as 168 horas de radiação ultravioleta. Isso faz com que a cor da madeira seja alterada com a participação dessa variável $\left(\mathrm{a}^{*}\right)$ como secundária. Certamente a variavel b* será a principal responsável da mudança da cor após o recebimento dos tratamentos estudados.

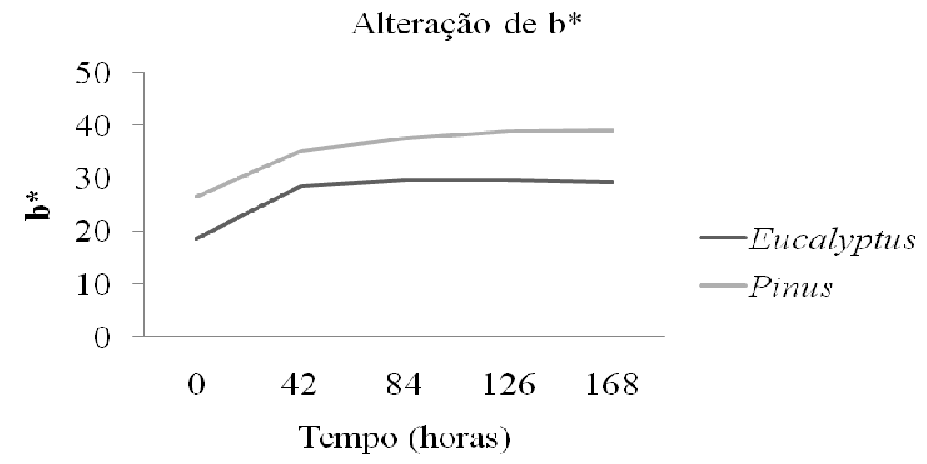

$$
\begin{gathered}
* \mathrm{~b}=18,76487+0,35121 \mathrm{~T}-0,003365 \mathrm{~T}^{2}+0,0000098 \mathrm{~T}^{3}, \mathrm{R}^{2}=0,98 \text {. Significativo a } 1 \% \text { (E. benthamii); } \\
* * \mathrm{~b}=27,20546+0,19051 \mathrm{~T}-0,000731 \mathrm{~T}^{2}, \mathrm{R}^{2}=0,95 \text {. Significativo a } 1 \% \text { (P. caribaea var. hondurensis) } .
\end{gathered}
$$

Figura 5. Alteração da coordenada $b^{*}$ em função da fotodegradação da madeira.

Figure 5. Change of coordinate $b^{*}$ according to photo degradation of wood.

A figura 5 mostra o comportamento colorimétrico das madeiras dado pela variável b*, em função da radiação ultravioleta nos tempos estudados. Verifica-se que a madeira de $P$. caribaea var. hondurensis apresentou valores maiores quando comparada com a madeira de E. benthamii, indicando a predominância da cor amarela para essa espécie. Observa-se, ainda, que a alteração da cor da madeira (coordenada $\mathrm{b}^{*}$ ) de E. benthamii apresenta um aumento até as 42 horas e posteriormente mostra uma tendência de estabilizar. A madeira de P.caribaea var. hondurensis também apresentou um aumento nas primeiras 42 horas, mostrando ainda, apesar de menos acentuada, uma tendência de continuar aumentando, parecendo estabilizar mais no final do último tratamento. Ou seja, a coordenada b* é a determinante na cor da madeira dessa espécie e o seu escurecimento é dado pela presença marcante dos componentes químicos que formam a cor amarela, que sofrem modificações pela ação da radiação ultravioleta.

\section{Reflectância e parâmetro $\Delta E$}

A madeira reflete a luz em intensidade diferente em cada comprimento de onda, de acordo com a sua coloração. Assim, pode-se dizer que cada espécie de madeira possui a sua assinatura espectral, na região do visível. Essa identidade da madeira se modifica em função de alterações sofridas em suas cores. Essa ferramenta é importante para verificar visualmente a alteração da cor da madeira, em cada comprimento de onda, em função da ação dos raios ultravioletas (GONÇALEZ et al., 2001).

Assim, observando-se as figuras 6 e 7, pode-se inferir que a madeira de E. benthamii apresentou uma menor claridade após exposições (42, 84, 126 e 168 horas), em função da diminuição da refletância. Esse mesmo comportamento também ocorreu na madeira de $P$. caribaea var. hondurensis, porém a diferença entre cor inicial (testemunha) e as cores após a radiação foi menor que a de E. benthamii, indicado pelas curvas de reflectâncias em cada tratamento.

Em ambas as espécies foi observado que as curvas correspondentes à madeira que recebeu radiação em diferentes tempos apresentaram, na região de 400 a $590 \mathrm{~nm}$, uma menor refletância, aumentando posteriormente, até atingirem a faixa do vermelho (acima de $620 \mathrm{~nm}$ ). Isso mostra a influência das pigmentações amarela e vermelha na formação da cor da madeira das duas espécies e a modificação da cor dada por essas pigmentações ao receberem irradiações ultravioletas. O escurecimento da madeira de E. benthamii é fortemente influenciado pela pigmentação vermelha, enquanto que na 
madeira de $P$. caribaea var. hondurensis, além da pigmentação vermelha, a amarela tem forte participação na formação da cor final pós-irradiação, explicando a coloração amarela "suja" resultante.

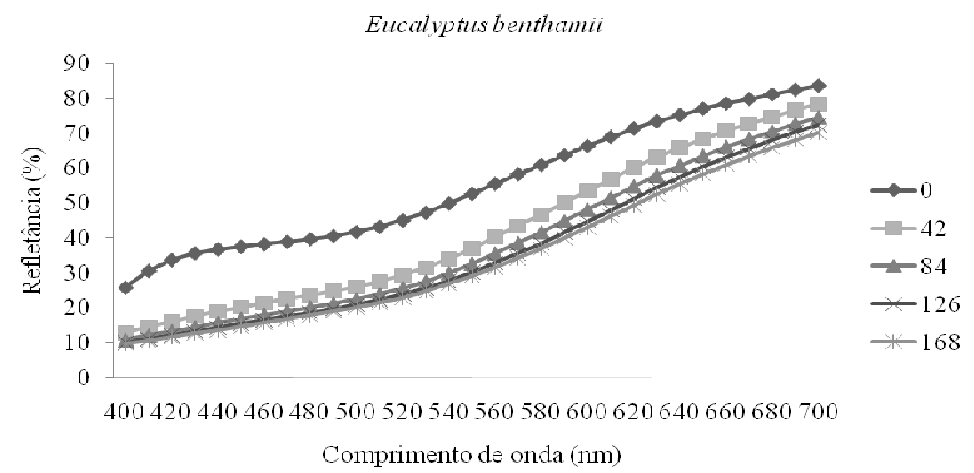

Figura 6. Refletância do Eucalyptus benthamii em diferentes tempos de exposição à irradiação ultravioleta.

Figure 6. Reflectance of Eucalyptus benthamii in different times of exposure to ultraviolet irradiation.

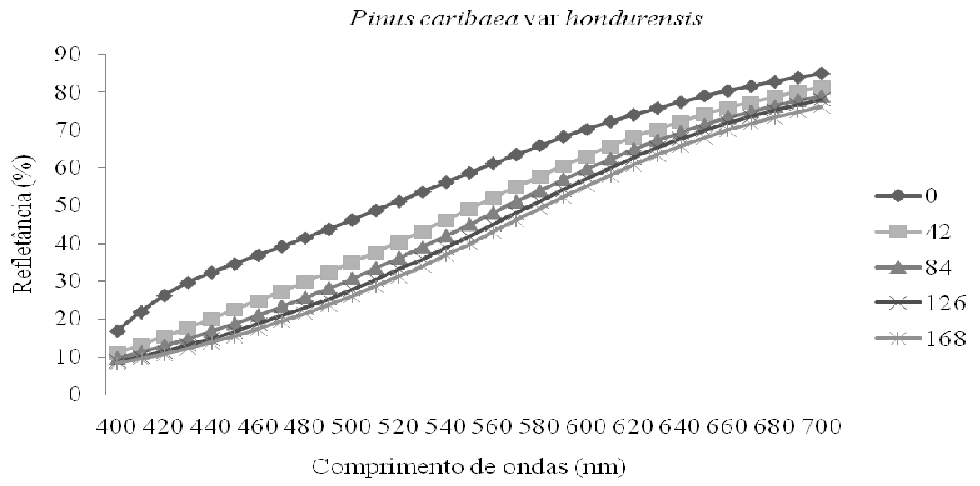

Figura 7. Refletância do P. caribaea var. hondurensis em diferentes tempos de exposição à irradiação ultravioleta.

Figure 7. Reflectance of $P$. caribaea var. hondurensis in different times of exposure to ultraviolet irradiation.

A figura 8 apresenta os valores médios encontrados para $\Delta \mathrm{E}$ nos diferentes tempos de radiação para as duas espécies estudadas. $\mathrm{O}$ parâmetro $\Delta \mathrm{E}$ é utilizado para determinar a alteração da cor da madeira e é calculado de acordo com a equação 1 , anteriormente citada.

Observa-se que a madeira de E. benthamii apresentou valores maiores de $\Delta \mathrm{E}$, sugerindo que ocorreu uma maior alteração na cor em comparação com a madeira de Pinus caribaea var. hondurensis, o que não era esperado, pois, segundo Mitsui; Tsuchikawa (2005), as madeiras de folhosas são consideradas mais resistentes à mudança de cor do que as madeiras de coníferas. Por outro lado, deve-se ressaltar que as amostras encontravam-se em diferentes planos de cortes. Segundo Gonçalez et al. (2006), a madeira apresenta coloração diferente de acordo com as faces de estudo.

Pode-se observar ainda que a alteração na cor foi maior nas primeiras 42 horas, apresentando $\Delta \mathrm{E}$ de 10,66 e 14,66 para $P$. caribaea var. hondurensis e E. benthamii, respectivamente. Posteriormente, nas 84 horas seguintes, o $P$. caribaea var. hondurensis apresentou $\Delta \mathrm{E}$ de 14,24 e o E. benthamii de 17,71, ou seja, um aumento menor em comparação ao encontrado na primeira exposição, e assim sucessivamente até as 168 horas de radiação. O mesmo foi encontrado por Tolvaj (1994), citado por Gouveia (2008), que, ao analisar a influência da radiação ultravioleta sobre a madeira de 16 espécies, concluiu que as modificações provocadas pela ação da radiação eram mais intensas durante as primeiras 20 horas de exposição, sendo nítido o escurecimento de todas as espécies. 


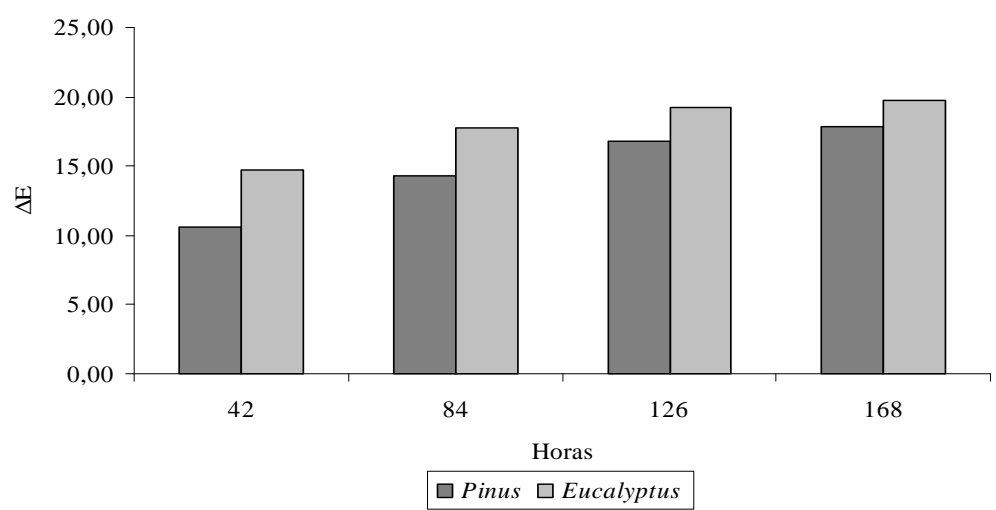

Figura 8. Parâmetro $\Delta \mathrm{E}$ para ambas as espécies nos diferentes tempos de irradiação ultravioleta.

Figure 8. Parameter $\Delta \mathrm{E}$ for both species in different times of exposure to ultraviolet irradiation.

\section{CONCLUSÕES}

- A técnica utilizada neste trabalho (envelhecimento artificial acelerado) permitiu simular uma avaliação da alteração da cor da madeira das espécies estudadas quando empregadas, por exemplo, em mobiliário e expostas em um ambiente natural de escritório.

- A exposição às radiações ultravioletas causou o escurecimento das madeiras de E. benthamii e de $P$. caribaea var. hondurensis, ocorrendo a maior alteração de cor nas primeiras horas de irradiação, confirmando assim a hipótese do trabalho.

- É importante a definição do plano de corte da madeira para o estudo do comportamento de sua cor. A madeira de $E$. benthamii em corte tangencial é mais susceptível à fotodegradação que a madeira de $P$. caribaea var. hondurensis em corte radial.

- Recomenda-se a continuação do estudo, utilizando-se produtos de acabamento de madeira. Essa técnica poderá contribuir na avaliação desses produtos perante a radiação ultravioleta com o passar do tempo.

\section{AGRADECIMENTOS}

Os autores expressam seus sinceros agradecimentos ao professor Ildeu Soares Martins, ao Laboratório de Produtos Florestais do Serviço Florestal Brasileiro, pela infraestrutura, e à KLABIN Unidade Otacílio Costa, pelo material fornecido.

\section{REFERÊNCIAS}

AYADI, N.; LEJEUNE, F.; CHARRIER, F.; CHARRIER, B.; MERLIN, A. Color stability of heattreated wood during artificial weathering. Holz als Roh-und Werkstoff, v. 61, n. 3, p. 221-226, jun. 2003.

CAMARGOS, J. A. A. Colorimetria quantitativa aplicada na elaboração de uma tabela de cores para madeiras tropicais. Brasília: UnB, 1999. 99 p. Dissertação (Mestrado) - Programa de PósGraduação em Ciências Florestais, Faculdade de Tecnologia, Universidade de Brasília, Brasília, 1999.

CAMARGOS, J. A. A.; GONÇALEZ, J. C. A colorimetria aplicada como instrumento na elaboração de uma tabela de cores de madeira. Brasil Florestal, Brasília, n. 71, p. 30-41, set. 2001. 
CHANG, H. T.; CHANG, S. T. Correlation between softwood discoloration induced by accelerated lightfastness testing and by indoor exposure. Polymer Degradation and Stability, v. 72, n. 2, p. 361365, may. 2001.

CHANG, S. T.; HON, S.; FEIST, W. C. Photodegradation and photoprotection of wood surfaces. Wood and fiber, v. 14, n. 2, p. 104-107, april. 1982.

CREEMERS, J.; MEIJER, M.; ZIMMERMANN, T.; SELL, J. Influence of climatic factors on the weathering of coated wood. Holz als Roh-und Werkstoff, v. 60, n. 6, p. 411-420, dec. 2002.

GEORGE, B.; SUTTIE, E.; MERLIN, A.; DEGLISE, X. Photodegradation and photostabilisation of wood - the state of the art. Polymer Degradation and Stability, v. 88, n. 2, p. 268-274, may. 2005.

GONÇALEZ, J. C.; JANIN, G.; SANTORO, A. C. S.; COSTA, A. F.; VALE, A. T. Colorimetria quantitativa: uma técnica objetiva de determinar a cor da madeira. Brasil Florestal, ano XX, n. 72, p. 4748, nov. 2001.

GONÇALEZ, J. C.; MARQUES, M. H. B.; OLIVEIRA, P. G.; JANIN, G.; CAMARGOS, J. A. A.; MARTINS, I. S. Caracterização das propriedades físicas e colorimétricas de duas espécies florestais para a indústria moveleira. In: $2^{\circ}$ Congresso Brasileiro de Industrialização da Madeira e Produtos de Base Florestal, Curitiba. CBIM 2006, v. 1, p. 1-8, 2006.

GOUVEIA, F. N. Aplicação de tratamentos térmicos para estabilização colorimétrica de madeiras tropicais. Brasília: UnB, 2008. 131 p. Tese (Doutorado) - Programa de Pós-Graduação em Ciências Florestais, Faculdade de Tecnologia, Universidade de Brasília, Brasília, 2008.

MITSUI, K.; TSUCHIKAWA, S. Low atmospheric temperature dependence on photodegradation of wood. Journal of Photochemistry and Photobiology B: Biology, v. 81, n. 2, p. 84-88, nov. 2005.

SUDIYANI, Y.; TSUJIYAMA, S.; IMAMURA, Y.; TAKAHASHI, M.; MINATO, K.; KAJITA, H. Chemical characteristics of surfaces of hardwood and softwood deteriorated by weathering. Journal of Wood Science, v. 45, n. 4, p. 348-353, aug. 1999.

TEMIZ, A.; YILDIZ, U. C.; AYDIN, I.; EIKENES, M.; ALFREDSEN, G.; ÇOLAKOGLU, G. Surface roughness and color characteristics of wood treated with preservatives after accelerated weathering test. Applied Surface Science, v. 250, p. 35-42, aug. 2005.

TSOUMIS, G. Science and technology of wood: structure, properties, utilization. New York: Chapman e Hall, 1991. 
